\title{
Impact of multi-gene mutational profiling on clinical trial outcomes in metastatic breast cancer
}

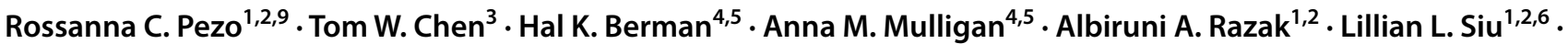 \\ David W. Cescon ${ }^{1,2} \cdot$ Eitan Amir $^{1,2} \cdot$ Christine Elser $^{1,2} \cdot$ David G. Warr $^{1,2} \cdot$ Srikala S. Sridhar $^{1,2} \cdot$ Celeste Yu ${ }^{6} \cdot$ Lisa Wang $^{7}$. \\ Tracy L. Stockley ${ }^{4,5,6} \cdot$ Suzanne Kamel-Reid ${ }^{4,5,6,8} \cdot$ Philippe L. Bedard ${ }^{1,2,6}[$
}

Received: 24 September 2017 / Accepted: 14 November 2017 / Published online: 24 November 2017

(c) The Author(s) 2017. This article is an open access publication

\begin{abstract}
Purpose Next-generation sequencing (NGS) has identified recurrent genomic alterations in metastatic breast cancer (MBC); however, the clinical utility of incorporating routine sequencing to guide treatment decisions in this setting is unclear. We examine the frequency of genomic alterations in MBC patients from academic and community hospitals and correlate with clinical outcomes.

Methods MBC patients with good performance status were prospectively recruited at the Princess Margaret Cancer Centre (PM) in Canada. Molecular profiling on DNA extracted from FFPE archival tissues was performed on the Sequenom MassArray platform or the TruSeq Amplicon Cancer Panel (TSACP) on the MiSeq platform. Clinical trial outcomes by RECIST 1.1 and time on treatment were reviewed retrospectively.

Results From January 2012 to November 2015, 483 MBC patients were enrolled and 440 were genotyped. At least one somatic mutation was identified in 46\% of patients, most commonly in PIK3CA (28\%) or TP53 (13\%). Of 203 patients with $\geq 1$ mutation(s), $15 \%$ were treated on genotype-matched and $9 \%$ on non-matched trials. There was no significant difference for median time on treatment for patients treated on matched vs. non-matched therapies (3.6 vs. 3.8 months; $p=0.89$ ). Conclusions This study provides real-world outcomes on hotspot genotyping and small targeted panel sequencing of MBC patients from academic and community settings. Few patients were matched to clinical trials with targeted therapies. More comprehensive profiling and improved access to clinical trials may increase therapeutic options for patients with actionable mutations. Further studies are needed to evaluate if this approach leads to improved clinical outcomes.
\end{abstract}

Keywords Molecular profiling $\cdot$ Metastatic breast cancer $\cdot$ Targeted therapies $\cdot$ PIK3CA mutation

Electronic supplementary material The online version of this article (https://doi.org/10.1007/s10549-017-4580-2) contains supplementary material, which is available to authorized users.

Philippe L. Bedard

philippe.bedard@uhn.ca

1 Division of Medical Oncology and Hematology, Princess Margaret Cancer Centre, 7-723 700 University Avenue, Toronto, Canada

2 Department of Medicine, University of Toronto, Toronto, Canada

3 Department of Oncology, National Taiwan University Hospital, Taipei, Taiwan

4 Laboratory Medicine Program, University Health Network, Toronto, Canada
5 Department of Laboratory Medicine and Pathobiology, University of Toronto, Toronto, Canada

6 Cancer Genomics Program, Princess Margaret Cancer Centre, Toronto, Canada

7 Department of Biostatistics, Princess Margaret Cancer Centre, Toronto, Canada

8 Department of Medical Biophysics, University of Toronto, Toronto, Canada

9 Present Address: Division of Medical Oncology and Hematology, Sunnybrook Odette Cancer Centre, Toronto, Canada 


\section{Introduction}

Mutational profiling of advanced solid tumors is an important component of early phase clinical trials testing drugs in molecularly defined patient populations. This approach, which currently involves the application of next-generation sequencing (NGS) technologies, is used to match a specific therapy to the particular somatic molecular alteration within a patient's tumor [1]. Although there is a variety of approved systemic therapies for metastatic breast cancer (MBC) that prolong progression-free or overall survival, including endocrine therapy, chemotherapy, and HER2-targeted therapy, $\mathrm{MBC}$ is an incurable disease and a leading cause of cancer death worldwide. The identification of subsets of breast cancers with overexpression of the HER-2/neu oncogene by IHC or amplification by fluorescence in situ hybridization (FISH) has led to the addition of targeted therapy for this subtype and improved survival [2, 3]. Additional molecular alterations such as somatic PIK3CA mutations, part of the PI3K/AKT/mTOR pathway, are commonly identified in breast cancers but are not yet used in the selection of approved therapies [4-7].

Integrated Molecular Profiling in Advanced Cancers Trial (IMPACT) and our community hospital program Community Oncology Molecular Profiling in Advanced Cancers Trial (COMPACT) were studies at Princess Margaret Cancer Centre (PM) to provide molecular profiling data for advanced solid tumor patients treated at PM and local community hospitals [8]. In this current study, we focus on molecular profiling in advanced breast cancers beyond standard estrogen receptor (ER), progesterone receptor (PR), and HER2 testing. We report on clinical characteristics, somatic mutation frequency, and therapeutic outcomes on genotype-matched and unmatched trials for MBC patients undergoing molecular sequencing of archival tumor tissues.

\section{Patients and methods}

\section{Study population}

Patients with histologically confirmed MBC were eligible for IMPACT/COMPACT if they were $\geq 18$ years, had Eastern Cooperative Oncology Group (ECOG) performance status $\leq 1$, and had available formalin-fixed paraffin-embedded (FFPE) archival tumor tissue (from either a primary or a metastatic site). This study was approved by the University Health Network Research Ethics Board and was registered on ClinicalTrials.gov [NCT01505400]. Enrollment for IMPACT began in March 2012 and for COMPACT in November 2012 and accrual to IMPACT/COMPACT ended in November 2015. For patients enrolled into clinical trials, the last follow-up was completed in March 2017 for this analysis.

\section{Tumor samples}

DNA was extracted from sections of the most recent FFPE tumor specimens available from biopsies or surgical resections. Optimal tumor regions were identified by clinical breast pathologists (AMM and HKB). Tumors containing a minimum acceptable tumor cellularity of $10 \%$ were processed with tumor regions isolated by $1-2 \times 1 \mathrm{~mm}$ punch from FFPE blocks or manual macrodissection of unstained material from 15 to 20 slides. FFPE samples were deparaffinized and treated with proteinase $\mathrm{K}$, followed by DNA extraction using the QIAmp DNA FFPE Tissue Kit (Qiagen, Germantown, MD) and quantification using the Qubit dsDNA Assay kit on the Qubit 2.0 Fluorometer (Thermo Fisher Scientific, Waltham, MA). DNA was extracted from peripheral blood samples for germline testing using either standard manual phenol/chloroform extraction methods or automated extraction (MagAttract DNA Mini M48 kit; Qiagen).

\section{Molecular profiling assays and PTEN testing}

Molecular profiling was performed in a College of American Pathologists (CAP) accredited and Clinical Laboratory Improvement Amendments (CLIA) certified laboratory. Details of the molecular profiling assays have been described in detail elsewhere [8]. Briefly, three molecular profiling assays were used over the study period: the TruSeq Amplicon Cancer Panel (TSACP, Illumina) on the MiSeq sequencer (Illumina) covering hotspot regions of 48 genes; the Ion AmpliSeq Cancer Panel (ASCP, Thermo Fisher Scientific) on the Ion Proton sequencer (Thermo Fisher Scientific) covering hotspot regions of 50 genes; and a custom multiplex genotyping panel on a matrix-assisted laser desorption/ionization time-of-flight (MALDI-TOF) massspectrometry platform (MassARRAY, Agena Bioscience, San Diego, CA) to genotype 279 mutations within 23 genes. Specific details of the sequencing panels used are shown in Supplementary Tables 1-3. FFPE samples tested by the TSACP and ASCP panels also had testing of matched blood samples for germline mutations. Sequence alignment, base calling, and variant assessment for the TSACP and ASCP panels were as previously described [8]. The scheme of Sukhai et al. [9] was used for assessment and classification of variants.

A subset of patients enrolled had testing for phosphatase and tensin homolog (PTEN) using immunohistochemistry (IHC) with rabbit monoclonal Ab 138G6 (Cell Signaling Technology, Danvers, MA) on a Dako platform using a dilution of 1:50 and Flex +30 protocol. Complete absence of 
tumor cell staining with positive staining of surrounding tumor stroma fibroblasts/endothelial cells was used to denote PTEN deficiency [10].

Molecular profiling results were included in the electronic medical record and returned to the treating oncologist. The clinical significance of profiling results was discussed with PM patients during a routine clinic visit by their treating oncologist and a PM oncologist reviewed the results with patients treated at other hospitals by telephone. Oncologists were provided with regular summary tables of testing results and mutation-specific clinical trial listings available at PM.

\section{Clinical data collection}

For each patient, baseline patient and tumor characteristics, treatment regimen(s), time on treatment(s), and survival were retrieved from medical records and updated every 3 months. Therapeutic clinical trial enrollment was evaluated from the date of reporting molecular profiling results until April 2016. Most recent follow-up for patients enrolled in clinical trial for this analysis was March 2017. Genotypematched clinical trials were defined as those restricting enrollment to patients with specific somatic mutations, those with a targeted drug with enriched clinical or preclinical activity in a patient's genotype, or those involving use of a drug that inhibited a pathway directly linked to the somatic mutation. Enrollment on clinical trial was based on trial availability and patient or physician preference and did not follow a pre-specified algorithm.

\section{End-points}

Radiological responses were defined as complete response (CR), partial response (PR), stable disease (SD), or progressive disease (PD) based on RECIST 1.1 criteria [11]. For comparison of clinical outcomes on matched versus unmatched therapies, therapeutic outcomes were evaluated according to time on treatment, defined as date of trial enrollment until date of discontinuation of investigational treatment.

\section{Statistical analysis}

Descriptive statistics were used to summarize patient characteristics, profiling results, and therapeutic activity. A generalized estimating equation model [12] was used to compare patients with profiling results treated on genotype-matched and genotype-unmatched trials. A mixed model was used to compare time on treatment, accounting for individual patients who were included on multiple therapeutic trials [13]. Differences with $p$ values of $<0.05$ were considered statistically significant.

\section{Results}

\section{Molecular profiling and PTEN results}

A total of 483 patients with MBC were enrolled from January 2012 to November 2015 as outlined in Fig. 1. Fifty-three percent of patients were registered by their treating oncologist at PM and $47 \%$ were referred from local community hospitals. Forty-three patients (9\%) who signed consent did not undergo profiling because of clinical deterioration before testing was performed or insufficient and/or poor quality tissue or DNA for sequencing analysis.

Of the 440 patients with genotyping results, 203 (46\%) had at least one mutation identified. The median followup time from date of profiling was 12.5 months (range 2 days-46 months). Table 1 lists the characteristics of patients who had molecular profiling performed on their tumors. The median age of all enrolled patients was 53 years (range 20-83 years) and median lines of systemic therapy (including endocrine treatment in hormone receptor-positive patients) received before enrolling into the molecular profiling program was 2 (range 1-18). Most patients had their primary tumor profiled $(n=326,74 \%)$. The most common histologies were ductal $(n=338,70 \%)$ and lobular carcinomas $(n=24,5 \%)$. Initial stage at diagnosis was either stage I or II in 155 patients (32\%), stage III in 76 patients (16\%), stage IV in 57 patients (12\%). Most tumors were grades 2 or $3(n=263,54 \%)$. Receptor status was ER positive/ HER-2 negative in 265 patients (55\%), HER-2 positive in 62 patients (13\%), and triple negative in 91 patients (19\%). Characteristics of patients with one or more mutations identified are also listed in Table 1.

As shown in Fig. 2, the most common somatic mutations were PIK3CA $(n=134,53 \%), \operatorname{TP} 53(n=81,32 \%)$, $\operatorname{PTEN}(n=9,3.5 \%)$, and $A K T 1(n=5,2 \%)$. Other genomic alterations were less common, including mutations in $K R A S$, ERBB2, BRAF, EGFR, SMAD4, FGFR1, FGFR2, FGFR3, $H R A S, N R A S, K D R$, and $C D H 1$. Details on the specific mutations identified in each patient are provided in Supplementary Table 4 . Figure 3 shows mutation frequencies detected by Sequenom versus TSACP/ASCP. Table 2 lists frequencies of mutations by receptor status of the tumor tissue used for molecular profiling. PIK3CA was the most common mutation identified in both ER positive/HER2 negative $(31 \%)$ and ER positive/HER2 positive $(64 \%)$ of tumors. TP53 mutations were the most commonly identified molecular alterations in triple-negative breast tissues at $41 \%$ and in ER negative/HER2 positive samples at 33\%. PTEN expression was tested by IHC and detected in 141 out of 163 patients $(87 \%)$. Twenty-two patients (14\%) had loss of PTEN in their tumors and 2 of these patients with PTEN deficiency (9\%) had tumors with somatic mutations in PIK3CA. 


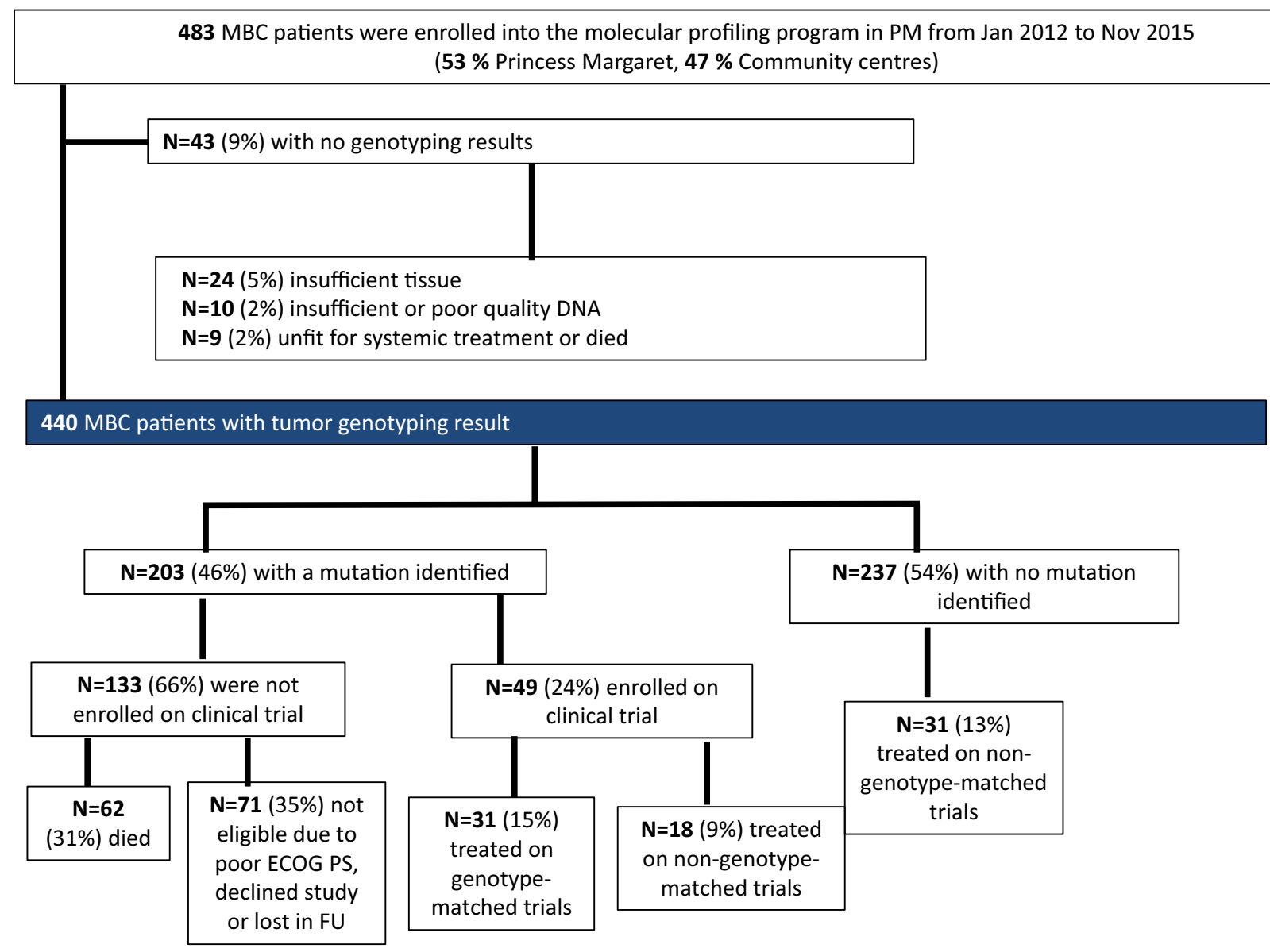

Fig. 1 Consort diagram of study schema and genotyping results

\section{Treatment for patients enrolled in clinical trials}

A total of 80 patients (18\%) with sequencing results were enrolled in therapeutic clinical trials at our institution, including 31 on genotype-matched trials and 49 on nongenotype-matched trials (Fig. 1). Of the 203 patients with at least one mutation, 49 (24\%) were enrolled in a therapeutic clinical trial after profiling results were reported, with 31/203 (15\%) enrolled in a genotype-matched trial and 18/203 (9\%) treated on non-matched trials (includes patients with actionable mutations not matched to a genotype-specific trial). Of 237 patients with no mutations identified, 31 $(13 \%)$ were enrolled in therapeutic trials after the date when molecular profiling results were reported.

The most common classes of drugs received on genotype-matched trials were PI3K inhibitors. Table 3 shows the somatic mutations identified and the best responses for patients treated on matched clinical trials. Radiological responses measured by RECIST 1.1 criteria are reported where available for patients treated on genotype-matched trials at our institution (including several patients enrolled in multiple matched trials). There was no significant difference for median time on treatment for patients treated on genotype-matched versus non-genotype-matched clinical trials (3.6 vs. 3.8 months; $p=0.89$ ). Response assessments were compared between genotype-matched and non-genotypematched patients enrolled in clinical trials as shown in Table 4. There were no significant differences in the best responses for patients enrolled in genotype-matched versus non-matched trials $(p=0.51)$.

\section{Discussion}

In this study, we identified one or more somatic mutations in $46 \%$ of patients with metastatic breast cancer using Sequenom hotspot or small targeted NGS panels. An important feature of our study is that we included patients receiving treatment in both academic and community settings that were referred for profiling while on standard of care therapies for metastatic disease. Albeit a non-randomized comparison, we did not observe a difference in time on treatment or best responses for profiled MBC patients treated on genotype-matched versus non-matched trials. 
Table 1 Baseline characteristics of all patients enrolled in the molecular profiling program

\begin{tabular}{|c|c|c|c|c|c|c|}
\hline \multirow[t]{2}{*}{ Characteristics } & \multicolumn{2}{|c|}{$\begin{array}{l}\text { All patients } \\
(n=483)\end{array}$} & \multicolumn{2}{|c|}{$\begin{array}{l}\text { Patients } \\
\text { with } \geq 1 \\
\text { mutation (s) } \\
(n=203)\end{array}$} & \multicolumn{2}{|c|}{$\begin{array}{l}\text { Patients with } \\
\text { no mutations } \\
(n=237)\end{array}$} \\
\hline & Number & $\%$ & Number & $\%$ & Number & $\%$ \\
\hline Median age (range) & $53(20-83$ & & & & & \\
\hline \multicolumn{7}{|l|}{ Histology } \\
\hline Ductal carcinoma & 338 & 70 & 152 & 75 & 186 & 78 \\
\hline Lobular carcinoma & 24 & 5 & 12 & 6 & 14 & 6 \\
\hline Mixed ductal and lobular carcinoma & 9 & 2 & 5 & 2 & 2 & 1 \\
\hline Other histology & 6 & 1 & 6 & 3 & 0 & 0 \\
\hline Invasive mammary carcinoma, type not defined & 64 & 13 & 28 & 14 & 35 & 15 \\
\hline Unknown & 42 & 9 & 0 & 0 & 0 & 0 \\
\hline \multicolumn{7}{|l|}{ Initial stage of diagnosis } \\
\hline $\mathrm{I} / \mathrm{II}$ & 155 & 32 & 73 & 36 & 79 & 33 \\
\hline III & 76 & 16 & 34 & 17 & 42 & 18 \\
\hline IV & 57 & 12 & 19 & 9 & 37 & 16 \\
\hline Unknown & 195 & 40 & 76 & 37 & 79 & 33 \\
\hline \multicolumn{7}{|l|}{ Tumor grade at diagnosis } \\
\hline 1 & 14 & 3 & 7 & 3 & 7 & 3 \\
\hline 2 and 3 & 263 & 54 & 116 & 57 & 142 & 60 \\
\hline Unknown & 206 & 43 & 77 & 38 & 88 & 37 \\
\hline Median lines of systemic treatment (range) & $2(1-18)^{\mathrm{a}}$ & & & & & \\
\hline \multicolumn{7}{|l|}{ Receptor status } \\
\hline ER positive/HER2 positive & 44 & 9 & 19 & 9 & 25 & 11 \\
\hline ER positive/HER2 negative & 265 & 55 & 112 & 55 & 153 & 65 \\
\hline ER negative/HER2 positive & 18 & 4 & 10 & 5 & 8 & 3 \\
\hline Triple negative & 91 & 19 & 52 & 26 & 39 & 16 \\
\hline ER positive/HER2 unknown & 10 & 2 & 5 & 2.5 & 5 & 2 \\
\hline Unknown & 55 & 11 & 5 & 2 & 7 & 3 \\
\hline \multicolumn{7}{|l|}{ Site of the sample used for genotyping $(n=440)$} \\
\hline Primary & 326 & 74 & 147 & 72 & 178 & 75 \\
\hline Metastasis & 114 & 26 & 56 & 28 & 59 & 25 \\
\hline \multicolumn{7}{|l|}{ Platform used for genotyping $(n=440)$} \\
\hline Princess margaret sequenom solid tumor panel & 317 & 72 & 59 & 29 & 155 & 65 \\
\hline $\begin{array}{l}\text { Illumina MiSeq TruSeq amplicon cancer panel/ } \\
\text { proton platform (TSACP/ASCP) }\end{array}$ & 123 & 28 & 139 & 71 & 83 & 35 \\
\hline
\end{tabular}

${ }^{\mathrm{a}}$ One patient received 18 lines of systemic therapies
An earlier study by Von Hoff and colleagues used IHC, FISH, and oligonucleotide microarrays for molecular profiling of tumor tissue from 66 patients (only 18 of whom had $\mathrm{MBC}$ ) with refractory metastatic disease to match patients to specific therapies and found a longer PFS on matched therapy compared to the patient's previous regimen [14]. However, the majority of MBC patients on this study received chemotherapy, hormonal or HER2-targeted therapies, not targeted treatments based upon identification of somatic mutations. Tsimberidou and colleagues used standard PCR-based sequencing to detect specific somatic genomic alterations in patients with refractory advanced malignancies and patients were enrolled in clinical trials according to genotype results [15]. Patients matched to targeted therapy had improved response rates, longer time to treatment failure (TTF), and longer survival than non-matched patients. However, only 16 patients in this study had MBC. In breast cancer-specific studies, SAFIR01 study used comparative genomic hybridization (CGH) array and Sanger sequencing to identify molecular alterations and match patients to targeted therapies [16]. Only 9\% of patients matched to targeted therapies had objective responses to treatment. In contrast to our study, where we used archival tissues for molecular testing, SAFIR required biopsy of the metastatic site if accessible. Also SAFIR limited enrollment to patients with no more 


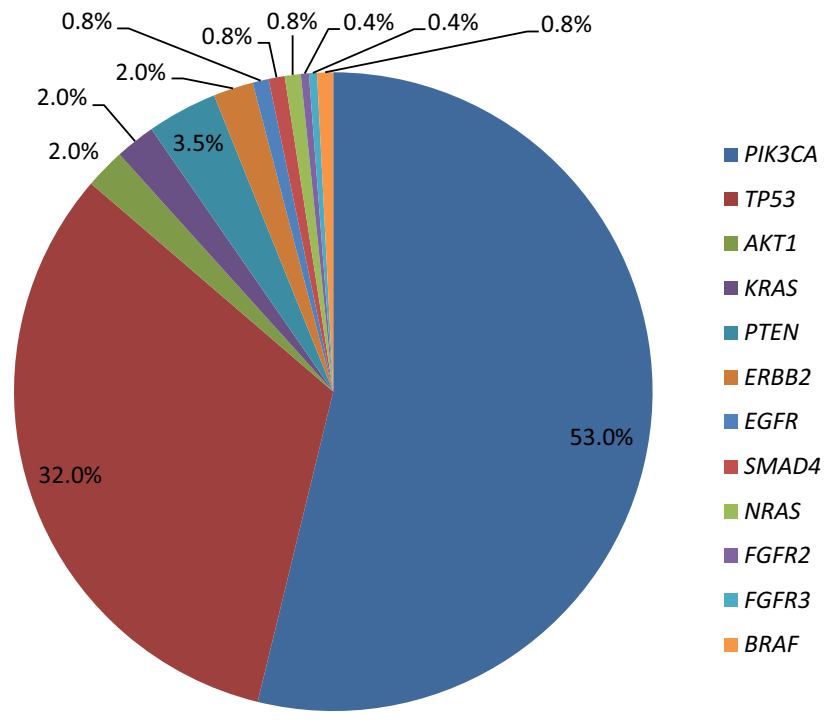

Fig. 2 Overall frequency (percentage) of somatic mutations out of 254 total mutations identified

than two prior lines of chemotherapy whereas our study did not have a limit on prior number of lines of chemotherapy.

More recent studies have used new technologies, such as NGS, to identify targetable molecular alterations. In the SHIVA trial, Le Tourneau and colleagues established molecular profiles for advanced solid tumors using targeted NGS, analysis of gene copy number alterations, and analysis of hormone receptor expression by IHC to match patients to targeted therapies [17]. This study differs from ours in several important aspects. The targeted agents given to the experimental group in SHIVA were drugs approved for clinical use but outside their approved indications, while in our study we matched patients to investigational drugs available through clinical trials at our institution. SHIVA required mandatory fresh biopsies whereas we tested archival samples.

In the MOSCATO 01 Trial, patients with advanced cancers were matched to targeted therapies based on molecular alterations and $33 \%$ were shown to have improved PFS outcomes with matched therapies when compared to PFS on prior therapy. Of the entire cohort, $19 \%$ of those matched to therapies were MBC patients. Molecular alterations were identified through array comparative genomic hybridization, NGS, and RNA sequencing performed on fresh frozen tumor biopsies [18]. MOSCATO 01 differs from our study in that they required fresh biopsies, which limited the patients enrolling in study to those willing to undergo invasive testing, those with tumors accessible for biopsy, and those willing to wait for the results of their molecular profiling for a decision on subsequent treatment. In our study patients had molecular profiling done on archival tissues while they were on systemic therapy and at the time of progression could be referred back for discussion of available targeted therapies on clinical trials based on identified genomic alterations.

In our study, the most common mutation identified among patients with genotyping results $(n=440)$ was in PIK3CA ( $n=123,28 \%$ ), which is consistent with other recent molecular profiling studies in MBC [16, 19-22]. Although mutations in PIK3CA are common in breast cancer, they have not been reliably predictive of clinical response to drugs targeting the PI3K/mTOR pathway [4-7]. PIK3CA mutations identified in archival tissues in BOLERO-2 [5], FERGI [23], BELLE-2 [24], and BELLE-3 [25] studies
Fig. 3 Overall frequency (percentage) of somatic mutations identified in all patients profiled $(n=440)$ on the Sequenom versus MiSeq/Proton platforms

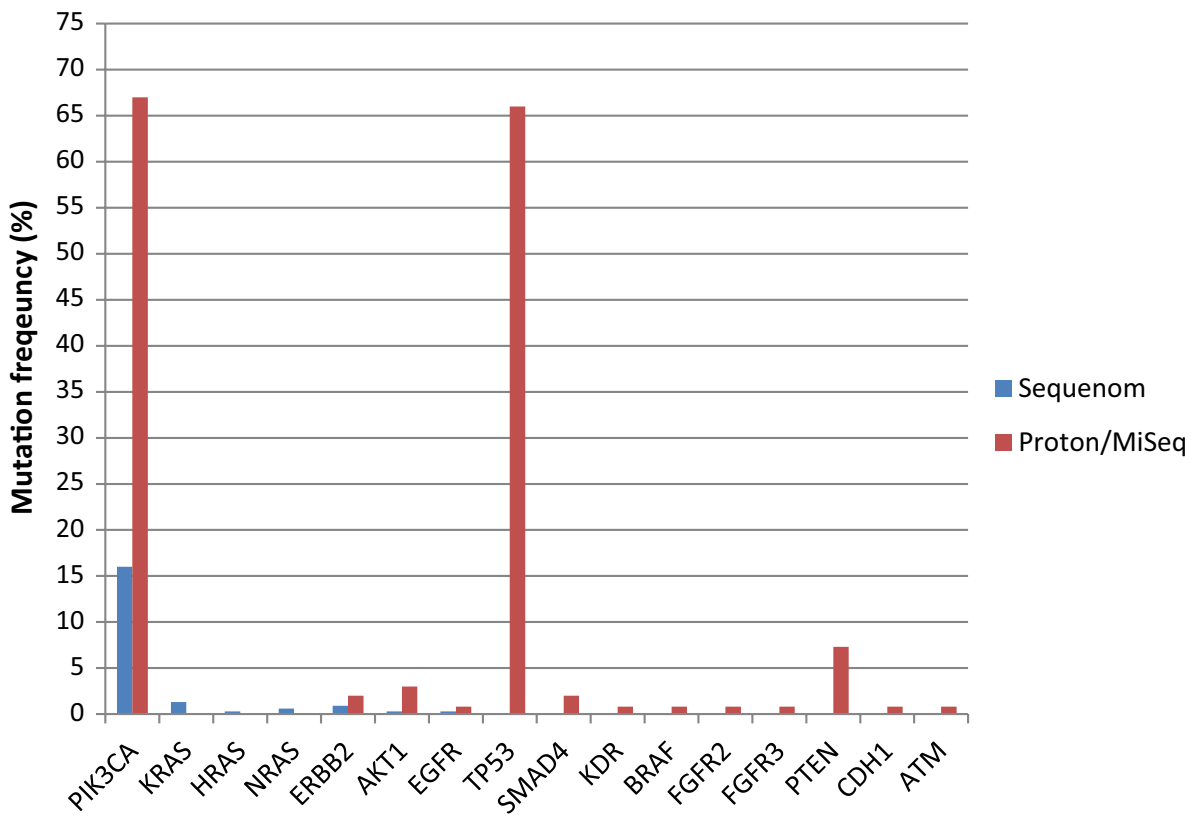


Table 2 Somatic mutation frequencies by receptor status of archival tissues used for molecular profiling

\begin{tabular}{|c|c|c|c|c|}
\hline \multirow[t]{2}{*}{ Gene } & \multicolumn{4}{|l|}{ Receptor status } \\
\hline & $\begin{array}{l}\text { ER+/HER2- } \\
n=265(\%)\end{array}$ & $\begin{array}{l}\text { ER+/HER2+ } \\
n=44(\%)\end{array}$ & $\begin{array}{l}\text { ER-/HER+ } \\
n=18(\%)\end{array}$ & $\begin{array}{l}\text { ER-/ } \\
\text { PR-/ } \\
\text { HER2- } \\
n=91 \\
(\%)\end{array}$ \\
\hline PIK3CA & $81(31)$ & $28(64)$ & $3(17)$ & 17 (19) \\
\hline TP53 & $20(8)$ & $16(36)$ & $6(33)$ & $37(41)$ \\
\hline PTEN & $3(1)$ & $2(5)$ & & $2(2)$ \\
\hline$A K T 1$ & $3(1)$ & & $1(6)$ & \\
\hline$B R A F$ & $1(0.4)$ & & & \\
\hline$E G F R$ & $2(0.8)$ & & & \\
\hline$E R B B 2$ & $3(1)$ & & $1(6)$ & \\
\hline$F G F R 2$ & $1(0.4)$ & & & \\
\hline$K R A S$ & $2(0.8)$ & & & $2(2)$ \\
\hline SMAD4 & $1(0.4)$ & & & $1(1)$ \\
\hline$K D R$ & & & & $1(1)$ \\
\hline
\end{tabular}

were not predictive of differential benefit with inhibitors of PI3K or mTOR. Thus in our study, where the majority of patients treated on genotype-matched trials were treated with PI3K inhibitors, the lack of significant differences in best responses and time on treatment may be reflective of the limited efficacy of PI3K inhibitors observed in PIK3CAmutant metastatic breast cancers.

As an alternative to tissue biopsies, identifying key mutations in circulating tumor DNA (ctDNA) in peripheral blood samples may provide more actionable information about the molecular profile of metastatic tumors. For example, in the BELLE-2 [24] and BELLE-3 [25] studies with the pan-PI3K inhibitor buparlisib combined with endocrine therapy, there was greater magnitude of effect on progression-free survival in the PIK3CA mutant subgroup identified through cell-free DNA but not in the PIK3CA mutant subgroup identified through archival tissue samples. However, in the BOLERO-2 study which examined the benefit of everolimus, an inhibitor of the PI3K/AKT/mTOR pathway, patients benefited from the addition of everolimus to endocrine therapy regardless of the presence of a PIK3CA mutation in either archival tissues [5] or cell-free DNA [26]. With novel isoform-selective PI3K inhibitors currently in testing clinical trials [27, 28], it remains to be determined whether PIK3CA mutation status tested using archival tissue samples or cell-free DNA is a biomarker of treatment response.

It is important to note that FERGI, BELLE-2, and BELLE-3 all involved pan-PI3K inhibitors, while the majority of patients treated on genotype-matched clinical trials at our institution were treated with isoform-selective PI3K inhibitors. More selective, isoform-specific PI3K inhibitors may be more effective and less toxic than pan-PI3K inhibitors, potentially leading to improved therapeutic outcomes [29]. However, the number of patients treated on isoform-selective versus pan-PI3K inhibitors is too small in our study to be able to make any conclusions regarding differential therapeutic outcomes.

TP53 was the second most common mutation identified in our breast cohort. The prevalence of TP53 mutations is lower in our cohort compared with other reports because TP53 mutation hotspots were not included in the Sequenom assay and the TSACP panel does not include full sequencing of the TP53 gene. TP53 mutations are associated with aggressive breast cancers and are identified in $>80 \%$ of basal-like breast cancer. Basho et al. analyzed archival tissues from 500 MBC patients including all subtypes using hotspot mutation testing and found that TP53 mutations were associated with worse clinical outcomes [22]. TP53 mutation is not currently a targetable genomic alteration, although inhibition of the protein kinase WEE1 in TP53-mutated cancers may be a potential future therapeutic approach [30,31]. Additionally more rare genomic alterations such as $A K T 1$ and ERBB2 mutations, each occurring in $2 \%$ of MBC patients in our cohort, are potential targets for AKT and ERBB2 inhibitors [32].

There are several important limitations to note in our study. First, we used archival tumor tissue which in some patients was many years removed from the date of study enrollment. Second, although our institution has a broad portfolio of early phase clinical trials, not all classes of targeted drugs were available during the course of the study for patients with actionable mutations. The majority of genotype-matched clinical trials available at our institution during the study period involved a PI3K inhibitor and most trials did not involve alpha isoform-selective/specific PI3K inhibitor that may have greater activity in PIK3CA mutant breast cancers $[33,34]$. Access to genotype-matched therapies for patients with actionable mutations was limited, and the clinical outcomes reported in our study may reflect the availability of effective targeted therapies and/or the usefulness of a sequencing-based treatment strategy. Available data do not allow for the exploration of the relative contribution of these limitations on our results. Access to a greater number of drugs targeting specific molecular alterations in basket trials, which enroll patients based on the molecular alteration of the tumor not on specific tumor types, such as the NCI-MATCH program [35], would broaden the availability of targeted drugs for actionable mutations. Unlike some other types of solid tumors, MBC patients have access to many lines of systemic therapy outside of clinical trials and some may have preferred other treatment options when invited to participate in a genotype-matched clinical trial. Only a small number of patients were enrolled in clinical trials following receipt of molecular profiling results and there was significant heterogeneity in the type of matched 
Table 3 Genotyping results and clinical trial agents for patients on matched trials

\begin{tabular}{|c|c|c|c|c|}
\hline $\begin{array}{l}\text { Site of sample used } \\
\text { for profiling }\end{array}$ & Mutations identified & Receptor status & Class of targeted drug received on clinical trials & Best response \\
\hline Primary lesion & $\mathrm{PIK} 3 \mathrm{CA} \gg \mathrm{N} 345 \mathrm{~K}$ & ER+/HER2- & Combination with PI3Ki & N/A \\
\hline Primary lesion & PIK3CA $\gg \mathrm{H} 1047 \mathrm{R}$ & Triple negative & Combination with PI3Ki & PR \\
\hline Primary lesion & PIK3CA 》 H1047R & ER+/HER2 unknown & Combination with PI3Ki & PD \\
\hline Primary lesion & PIK3CA 》 E545 K & ER+/HER2- & $\mathrm{PI} 3 \mathrm{Ki}$ & $\mathrm{SD}$ \\
\hline Primary lesion & PIK3CA 》 E542 K & Triple negative & Combination with PI3Ki & PR \\
\hline Primary lesion & PIK3CA $\gg \mathrm{H} 1047 \mathrm{R}$ & ER+ HER2+ & Combination with PI3Ki & PD \\
\hline Primary lesion & PIK3CA $\gg \mathrm{H} 1047 \mathrm{R}$ & ER+/HER2- & $\mathrm{PI} 3 \mathrm{Ki}$ & PR \\
\hline Primary lesion & PIK3CA 》 H1047R & $\mathrm{ER}+\mathrm{HER} 2+$ & AKTi & PD \\
\hline Metastatic lesion & $\begin{array}{l}\text { PIK3CA } \gg Q 545 G \\
\text { PTEN } \gg \text { L320X } \\
\text { PIK3CA } \gg \text { R93Q }\end{array}$ & ER+/HER2- & AKTi & PD \\
\hline Metastatic lesion & PIK3CA 》 E545 K & ER+/HER2- & $\mathrm{PI} 3 \mathrm{Ki}$ & N/A \\
\hline Primary lesion & $\begin{array}{l}\text { PIK3CA } \gg \mathrm{H} 1047 \mathrm{R} \\
\text { TP53 } \gg \mathrm{P} 278 \mathrm{~S}\end{array}$ & ER+/HER2- & $\mathrm{PI} 3 \mathrm{Ki}$ & SD \\
\hline Primary lesion & PIK3CA $\gg \mathrm{H} 1047 \mathrm{R}$ & ER+/HER2- & $\mathrm{PI} 3 \mathrm{Ki}$ & PR \\
\hline Metastatic lesion & PIK3CA 》 E542 K & ER+/HER2- & $\mathrm{PI} 3 \mathrm{Ki}$ & N/A \\
\hline Metastatic lesion & PIK3CA $\gg \mathrm{H} 1047 \mathrm{R}$ & ER+/HER2- & $\mathrm{PI} 3 \mathrm{Ki}$ & N/A \\
\hline Primary lesion & $\mathrm{PIK} 3 \mathrm{CA} \gg \mathrm{N} 345 \mathrm{~K}$ & ER+/HER2- & Combination with $\mathrm{PI} 3 \mathrm{Ki}$ & PD \\
\hline Primary lesion & $\begin{array}{l}\text { PIK3CA } \gg \mathrm{N} 345 \mathrm{~K} \\
\text { NRAS } \gg \mathrm{G} 12 \mathrm{D}\end{array}$ & $\begin{array}{l}\text { ER+/HER2- } \\
\text { ER+/HER2- }\end{array}$ & $\begin{array}{l}\text { Combination with PI3Ki } \\
\text { Combination with PI3Ki and FGFRi } \\
\text { PI3Ki alone }\end{array}$ & $\begin{array}{l}\mathrm{PD} \\
\mathrm{PD} \\
\mathrm{PR}\end{array}$ \\
\hline Primary lesion & PIK3CA $\gg \mathrm{H} 1047 \mathrm{~L}$ & ER+/HER2- & Combination PI3Ki & PD \\
\hline Primary lesion & PIK3CA $\gg$ E542 K & ER+/HER2- & $\mathrm{PI} 3 \mathrm{Ki}$ & PD \\
\hline Primary lesion & $\begin{array}{l}\text { PIK3CA } \gg \text { c. } 1633 \mathrm{G}>\mathrm{A} \\
\quad \text { (p.Glu545Lys) }\end{array}$ & $\mathrm{ER}+/ \mathrm{HER} 2-$ & AKTi & SD \\
\hline Primary lesion & $\begin{array}{l}\text { PIK3CA } \gg \mathrm{E} 542 \mathrm{~K} \\
\mathrm{ERBB} 2 \gg \mathrm{L} 755 \mathrm{~S}\end{array}$ & ER+/HER2- & HER2 TKI & PD \\
\hline Primary lesion & $\begin{array}{l}\text { PIK3CA } \gg \mathrm{E} 545 \mathrm{~K} \\
\text { TP53 } \gg \mathrm{L} 252 \mathrm{del} \\
\mathrm{BRAF} \gg \mathrm{c} .1315-4 \mathrm{C}>\mathrm{G}\end{array}$ & ER-/HER2+ & Combination with PI3Ki & SD \\
\hline Primary lesion & $\mathrm{PIK} 3 \mathrm{CA} \gg \mathrm{N} 345 \mathrm{~K}$ & ER+/HER2- & $\mathrm{PI} 3 \mathrm{Ki}$ & SD \\
\hline Primary lesion & PIK3CA $\gg \mathrm{H} 1047 \mathrm{E}$ & ER+/HER2- & $\mathrm{PI} 3 \mathrm{Ki}$ & SD \\
\hline Primary lesion & $\begin{array}{l}\mathrm{PIK} 3 \mathrm{CA} \gg \mathrm{H} 1047 \mathrm{~L} \\
\text { TP53 } \gg \mathrm{C} 238 \mathrm{Y}\end{array}$ & ER-/HER+ & Combination with PI3Ki and EGFRi & SD \\
\hline Primary lesion & PIK3CA 》 H1047R & $\mathrm{ER}+/ \mathrm{HER}-$ & $\mathrm{PI} 3 \mathrm{Ki}$ & N/A \\
\hline Primary lesion & PIK3CA $\gg \mathrm{H} 1047 \mathrm{~L}$ & ER+/HER2- & PI3Ki & SD \\
\hline Metastatic lesion & $\begin{array}{l}\text { PIK3CA 》 H1047R } \\
\text { TP53 》R248Q }\end{array}$ & ER-/HER- & PI3Ki & SD \\
\hline Primary lesion & 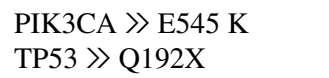 & ER+/HER2- & $\mathrm{PI} 3 \mathrm{Ki}$ & SD \\
\hline Metastatic lesion & $\begin{array}{l}\mathrm{ERBB} 2 \gg \mathrm{D} 769 \mathrm{H} \\
\text { PIK3CA } \gg \mathrm{N} 345 \mathrm{~K}\end{array}$ & ER+/HER2- & $\begin{array}{l}\text { HER2 TKI } \\
\text { PI3Ki }\end{array}$ & $\begin{array}{l}\text { SD } \\
\text { SD }\end{array}$ \\
\hline Primary lesion & FGFR2 $\gg \mathrm{Y} 376 \mathrm{C}$ & ER+/HER2- & FGFRi & $\mathrm{SD}$ \\
\hline
\end{tabular}

Best responses according to RECIST 1.1 criteria are listed if available. One patient was enrolled in two different clinical trials with PI3K inhibitors

N/A not available, $P R$ partial response, $P D$ progressive disease, $S D$ stable disease, $P I 3 K$ phosphatidylinositol 3-kinase, $m T O R$ mammalian target of rapamycin, $A K T$ protein kinase B, EGFR epidermal growth factor receptor, TKI tyrosine-kinase inhibitor, FGFR fibroblast growth factor receptor 
Table 4 Comparison of best responses for patients on genotypematched versus non-genotype-matched clinical trials

\begin{tabular}{llll}
\hline Best response & Trial enrollment & Total \\
\cline { 2 - 3 } & $\begin{array}{l}\text { Non-genotype- } \\
\text { matched }\end{array}$ & $\begin{array}{l}\text { Genotype- } \\
\text { matched }\end{array}$ & \\
\hline No response & 55 & 27 & 82 \\
Response & 6 & 5 & 11 \\
Total & 61 & 32 & 93 \\
\hline
\end{tabular}

Response data not available for all patients. Includes two patients enrolled in multiple trials

drug treatments received. There were also limitations in our sequencing approach. We used small hotspot genotyping or targeted sequencing panels for frequent point mutations and insertions/deletions only. While these capture the majority of somatic alterations in breast cancer, we did not assess gene amplifications or fusions which are potential genomic driver or tumor suppressor alterations that are relevant for genotype-matched treatment selection, such FGFRI amplification or fusions, $B R C A 1 / 2$ mutations, NTRK fusions, $P I K 3 C A$ fusions and ERBB2 fusions [36].

In conclusion, our data provide "real-world" clinical outcomes for patients with MBC who have undergone molecular profiling with hotspot genotyping or small targeted NGS testing. We found that only a small percentage of patients with MBC profiled using hotspot genotyping or small targeted NGS testing subsequently enrolled in genotypematched clinical trials. In this non-randomized comparison, we did not observe a difference in time of treatment for patients subsequently enrolled in genotype-matched versus non-genotyped-matched clinical trials. Further studies are required to establish the clinical utility of routine multi-gene mutation testing for patients with MBC.

Acknowledgements The authors acknowledge all the patients, medical oncologists, pathologists, laboratory technicians, clinical data coordinators, and correlative studies coordinators who participated in this research study. This work was supported by the Princess Margaret Cancer Foundation; the Cancer Care Ontario Applied Research Unit [to LLS]; the University of Toronto Division of Medical Oncology Strategic Innovation [to PLB]; and the Ontario Ministry of Health and Long-Term Care Academic Health Sciences Centre Alternate Funding Plan Innovation Award [to PLB].

\section{Compliance with ethical standards}

Conflict of interest No relevant conflicts of interest to disclose.

Ethical standards This study was approved by the University Health Network Research Ethics Board.

Informed consent Informed consent was obtained for all participants in this study.
Open Access This article is distributed under the terms of the Creative Commons Attribution 4.0 International License (http://creativecommons.org/licenses/by/4.0/), which permits unrestricted use, distribution, and reproduction in any medium, provided you give appropriate credit to the original author(s) and the source, provide a link to the Creative Commons license, and indicate if changes were made.

\section{References}

1. Meric-Bernstam F, Brusco L, Shaw K et al (2015) Feasibility of large-scale genomic testing to facilitate enrollment onto genomically matched clinical trials. J Clin Oncol 33:2753-2762

2. Slamon DJ, Leyland-Jones B, Shak S et al (2001) Use of chemotherapy plus a monoclonal antibody against HER2 for metastatic breast cancer that overexpresses HER2. N Eng1 J Med 344(11):783-792

3. Swain SM, Baselga J, Kim SB et al (2015) Pertuzumab, trastuzumab, and docetaxel in HER2-positive metastatic breast cancer. N Engl J Med 372(8):724-734

4. Juric D, Baselga J (2012) Tumor genetic testing for patient selection in phase I clinical trials: the case of PI3K inhibitors. J Clin Oncol 30(8):765-766

5. Hortobagyi GN, Chen D, Piccart M et al (2016) Correlative analysis of genetic alterations and everolimus benefit in hormone receptor-positive, human epidermal growth factor receptor 2-negative advanced breast cancer: results from BOLERO-2. J Clin Oncol 34(5):419-426

6. Al-Suksun S, Lataifeh I, Al-Sukhun R (2016) Defining the prognostic and predictive role of PIK3CA mutations: shifting through the conflicting data. Curr Breast Cancer Rep 8(2):73-79

7. Mukohara T (2015) PI3 K mutations in breast cancer: prognostic and therapeutic implications. Breast Cancer (Dove Med Press) 7:111-123

8. Stockley TL, Oza AM, Berman HK et al (2016) Molecular profiling of advanced solid tumors and patient outcomes with genotype-matched clinical trials: the princess margaret IMPACT/ COMPACT trial. Genomics Med 8(106):1-12

9. Sukhai MA, Craddock KJ, Thomas M et al (2016) A classification system for clinical relevance of somatic variants identified in molecular profiling of cancer. Genet Med 18(2):128-136

10. Yanagawa N, Leduc C, Kohler D et al (2012) Loss of phosphatase and tensin homolog protein expression is an independent poor prognostic marker in lung adenocarcinoma. J Thorac Oncol 7(10):1513-1521

11. Eisenhauer EA, Therasse P, Bogaerts J et al (2009) New response evaluation criteria in solid tumours: revised RECIST guideline (version 1.1). Eur J Cancer 45(2):228-247

12. Touloumis A, Agresti A, Kateri M et al (2013) GEE for multinomial responses using a local odds ratios parameterization. Biometrics 69(3):633-640

13. Therneau TM, Grambsch PM (2000) Modeling survival data: extending the Cox model. Springer, New York

14. Von Hoff DD, Stephenson JJ Jr, Rosen P et al (2010) Pilot study using molecular profiling of patients' tumors to find potential targets and select treatments for their refractory cancers. J Clin Oncol 28(33):4877-4883

15. Tsimberidou AM, Iskander NG, Hong DS et al (2012) Personalized medicine in a phase I clinical trials program: the MD Anderson Cancer Center initiative. Clin Cancer Res 18(22):6373-6383

16. Andre F, Bachelot T, Commo F et al (2014) Comparative genomic hybridisation array and DNA sequencing to direct treatment of metastatic breast cancer: a multicentre, prospective trial (SAFIR01/UNICANCER). Lancet Oncol 15(3):267-274 
17. Le Tourneau C, Delord JP, Goncalves A et al (2015) Molecularly targeted therapy based on tumour molecular profiling versus conventional therapy for advanced cancer (SHIVA): a multicentre, open-label, proof of concept, randomized, controlled phase 2 trial. Lancet Oncol 16(13):1324-1334

18. Massard C, Michiels S, Ferte C et al (2017) High-throughput genomics and clinical outcome in hard-to-treat advanced cancers: results of the MOSCATO 01 trial. Cancer Discov 7(6):1-10

19. Cejalvo JM, Perez-Fidalgo JA, Ribas G et al (2016) Clinical implications of routine genomic mutation sequencing in PIK3CA/ AKT1 and KRAS/NRAS/BRAF in metastatic breast cancer. Breast Cancer Res Treat 160(1):69-77

20. Vasan NR, Yelensky R, Wang K et al (2014) A targeted nextgeneration sequencing assay detects a high frequency of therapeutically targetable alterations in primary and metastatic breast cancers: implications for clinical practice. Oncologist 19(5):453-458

21. Muller KE, Marotti JD, de Abreu FB et al (2016) Targeted nextgeneration sequencing detects a high frequency of potentially actionable mutations in metastatic breast cancers. Exp Mol Pathol 100(3):421-425

22. Basho RK, Gagliato DM, Ueno NT et al (2016) Clinical outcomes based on multigene profiling in metastatic breast cancer patients. Oncotarget 7(47):76362-76373

23. Krop IE, Mayer I, Ganju V et al (2016) Pictilisib for oestrogen receptor-positive, aromatase inhibitor-resistant, advanced or metastatic breast cancer (FERGI): a randomized, double-blind, placebo-controlled, phase 2 trial. Lancet Oncol 17:811-821

24. Baselga J, Iwata $\mathrm{H}$, Clemons $\mathrm{M}$ et al (2015) PIK3Ca status in circulating tumor DNA (ctDNA) predicts efficacy of buparlisib (BUP) plus fulvestrant (FULV) in postmenopausal women with endocrine-resistant HR+/HER2- advanced breast cancer (BC): first results from the randomized, phase III BELLE-2 trial. Presented at the San Antonio Breast Cancer symposium 2015, Abstract S6-01

25. Di Leo A., Seok LK, Ciruelos E et al (2016) BELLE-3: a phase III study of buparlisib + fulvestrant in postmenopausal women with HR+, HER2-, aromatase inhibitor-treated, locally advanced or metastatic breast cancer, who progressed on or after mTOR inhibitor-based treatment. Presented at San Antonio Breast Cancer Symposium 2016, abstract S4-07

26. Moynahan ME, Chen D, He W et al (2017) Correlation between PIK3CA mutations in cell-free DNA and everolimus efficacy in HR+, HER2 - advanced breast cancer: results from BOLERO-2. Br J Cancer 116(6):726-730

27. Fritsch C, Huang A, Chatenay-Rivauday C et al (2014) Characterization of the novel and specific PI3 K $\alpha$ Inhibitor NVP-BYL719 and development of the patient stratification strategy for clinical trials. Mol Cancer Ther 13(5):1117-1129

28. Olivero AG, Heffron TP, Baumgardner M et al (2013) Discovery of GDC-0032: A beta-sparing PI3K inhibitor active against PIK3CA mutant tumors. Cancer Res 73(8 Suppl):Abstract nr DDT02-01

29. Thorpe LM, Yuzugullu H, Zhao JJ (2015) PI3 K in cancer: divergent roles of isoforms, mode of activation and therapeutic targeting. Nat Rev Cancer 15:7-24

30. Aarts M, Sharpe R, Garcia-Murillas I et al (2012) Forced mitotic entry of S-phase cells as a therapeutic strategy induced by inhibition of WEE1. Cancer Discov 2(6):524-539

31. Bauman JE, Chung CH (2014) CHK it Out! Blocking WEE kinase routs TP53 mutant cancer. Clin Cancer Res 20(16):4173-4175

32. Carmona FJ, Montemurro F, Kannan S et al (2015) AKT signaling in ERBB2-amplified breast cancer. Pharmacol Ther 158:63-70

33. Dickler MN, Saura C, Richards D et al (2016) A Phase II study of the PI3K inhibitor taselisib (GDC-0032) combined with fulvestrant in patients with HER2-, hormone receptor-positive advanced breast cancer. J Clin Oncol 34(15):520

34. Mayer IA, Abramson VG, Formisano L et al (2017) A Phase Ib study of alpelisib (BYL719), a PI3K $\alpha$-specific inhibitor, with letrozole in ER+/HER2- metastatic breast cancer. Clin Cancer Res 23(1):26-34

35. Conley BA, Doroshow JH (2014) Molecular Analysis for therapy of choice: NCI MATCH. Semin Oncol 41(3):297-299

36. Paratala BS, Dolfi SC, Khiabanian $\mathrm{H}$ et al (2016) Emerging role of genomic rearrangments in breast cancer: applying knowledge from other cancers. Biomarkers in Cancer 8(S1):1-14 